\title{
Abordagem Simplificada do Nervo Ciático por Via Posterior, no Ponto Médio do Sulco Glúteo-Femoral, com Uso de Neuroestimulador *
}

\section{Simplified Sciatic Nerve Approach by the Posterior Route at the Median Gluteus-Femoral Sulcus Region, with a Neurostimulator}

Neuber Martins Fonseca, TSA ${ }^{1}$, Fernando Xavier Ferreira ${ }^{2}$, Roberto Araújo Ruzi ${ }^{2}$, Gulherme Carnaval Souza Pereira ${ }^{2}$

\begin{abstract}
RESUMO
Fonseca NM, Ferreira FX, Ruzi RA, Pereira GCS - Abordagem Simplificada do Nervo Ciático por Via Posterior, no Ponto Médio do Sulco Glúteo-Femoral, com Uso de Neuroestimulador
\end{abstract}

\begin{abstract}
Justificativa e Objetivos - O bloqueio do nervo ciático pode ser realizado por várias abordagens com vantagens e desvantagens. O nervo ciático é o maior nervo do corpo humano em diâmetro e comprimento. É a continuação do fascículo superior do plexo sacral $\left(L_{4}, L_{5}, S_{1}, S_{2}\right.$ e $\left.S_{3}\right)$. Sai da pelve através do forâmen isquiático maior, passando por baixo do músculo piriforme, desce entre o trocânter maior do fêmur e a tuberosidade isquiática, e ao longo do dorso da coxa, anterior aos músculos bíceps femoral e semitendinoso, até o terço inferior da coxa, onde se divide em dois grandes ramos denominados nervos tibial e fibular comum. Torna-se superficial na borda inferior do músculo glúteo máximo. Baseados nesta descrição anatômica, desenvolvemos uma abordagem posterior, tendo como vantagens a fácil identificação da anatomia de superfície, superficialidade do nervo nesta localização, provocando menor desconforto ao paciente que outras abordagens e podendo utilizar agulha de $5 \mathrm{~cm}$.
\end{abstract}

Método - Foram estudados 17 pacientes, estado físico ASA I, II ou III, com idades entre 21 e 79 anos, peso de 55 a $90 \mathrm{~kg}$, submetidos a cirurgias em perna ou pé. Após monitorização, o paciente foi posicionado em decúbito ventral e realizado bloqueio no ponto médio do sulco glúteo-femoral (dobra da pele entre a nádega e região posterior da coxa), com auxílio de neuroestimulador, utilizando lidocaína a $1 \%$ sem adrenalina (300 mg). Avaliou-se latência, tempo de execução do bloqueio, anestesia dos nervos tibial, fibular comum e cutâneo posterior da coxa. Quando necessário, foi também realizado o bloqueio do nervo safeno com $5 \mathrm{ml}$ de lidocaína a $1 \%$.

Resultados - Obteve-se anestesia adequada em todos os casos com o volume e concentração usados. Em nenhum

Recebido do (Received from) Serviço de Anestesiologia da Faculdade de Medicina da Universidade Federal de Uberlândia (FM-UFU), Uberlândia, MG

1. Professor Adjunto da Disciplina de Anestesiologia; Responsável pelo CET/SBA da FM-UFU e Membro da Comissão de Normas Técnicas da Sociedade Brasileira de Anestesiologia

2. Anestesiologista do Serviço de Anestesiologia (SEANE) do Hospital de Clínicas da Faculdade de Medicina da Universidade Federal de Uberlândia

Apresentado (Submitted) em 06 de fevereiro de 2002

Aceito (Accepted) para publicação em 05 de abril de 2002

Correspondência para (Mail to):

Dr. Neuber Martins Fonseca

Rua Antonio Luis Bastos, 300 - Altamira II

38411-116 Uberlândia, MG

E-mail: neuber@triang.com.br

(C) Sociedade Brasileira de Anestesiologia, 2002 paciente ocorreu anestesia do nervo cutâneo posterior da coxa. O tempo de execução do bloqueio foi de 8,58 \pm 5,71 min. A latência foi de 5,88 $\pm 1,6 \mathrm{~min}$. A duração sensitiva e motora do bloqueio foi de 4,05 \pm 1,1 e 2,9 $9 \pm 0,8$ horas, respectivamente.

Conclusões - Essa nova abordagem é eficaz e de fácil execução. Não está indicada se o bloqueio do nervo cutâneo posterior da coxa for necessário.

UNITERMOS: ANATOMIA: nervo ciático. ANESTÉSICOS, Local: lidocaína; TÉCNICAS ANESTÉSICAS, Regional: nervos periféricos, ciático

\section{SUMMARY}

Fonseca NM, Ferreira FX, Ruzi RA, Pereira GCS - Simplified Sciatic Nerve Approach by the Posterior Route at the Median Gluteus-Femoral Sulcus Region, with a Neurostimulator

Background and Objectives - The sciatic nerve may be blocked by several routes, all of them with advantages and disadvantages. It is the largest human nerve in diameter and length, being the prolongation of the upper sacral plexus fascicle $\left(L_{4}, L_{5}, S_{2}\right.$ and $\left.S_{3}\right)$. It leaves the pelvis through the foramen ischiadicum majus, passing below the piriform muscle and going down between the greater trochanter and the ischial tuberosity, continuing along the femoral dorsum, anterior to biceps femoris and semitendinous muscles, to the lower femoral third, where it is divided in two major branches called tibial and common fibular nerves. It becomes superficial at the lower border of the gluteus maximus muscle. Based on this anatomic description, we developed a posterior approach with the following advantages: easy identification of the surface anatomy, superficial level of the nerve at this location; and less discomfort to patients since a $5 \mathrm{~cm}$ needle may be used.

Methods - Seventeen ASA I - III patients aged 21 to 79 years, weighing 55 to $90 \mathrm{~kg}$, undergoing leg or foot surgery were studied. After monitoring, patients were placed in the prone position and blockade was performed at the middle point of the sulcus gluteus (skin fold between nates and posterior thigh), with the aid of a neurostimulator, using $1 \%$ plain lidocaine $(300 \mathrm{mg})$. Onset time, blockade performing time, and tibial, common fibular and cutaneous femoris posterior nerves anesthesia were evaluated. Saphenous nerve was also blocked with $5 \mathrm{ml}$ of $1 \%$ lidocaine whenever needed.

Results - Adequate anesthesia was obtained in all cases. There was no patient with cutaneous femoris posterior nerve anesthesia. Blockade performing time was $8.58 \pm 5.71 \mathrm{~min}$. Onset time was $5.88 \pm 1.6 \mathrm{~min}$. Sensory and motor block duration was $4.05 \pm 1.1$ and $2.9 \pm 0.8$ hours, respectively.

Conclusions - This new approach is effective and easy. However, it is not indicated when the cutaneous femoris posterior nerve anesthesia is necessary.

KEY WORDS: ANATOMY: sciatic nerve. ANESTHETICS, Local: lidocaine; ANESTHETIC TECHNIQUES, Regional: peripheral nerve, sciatic 


\section{INTRODUÇÃO}

Acombinação do bloqueio do nervo ciático e do 3 em 1 é satisfatória para pacientes submetidos à cirurgia de membros inferiores, podendo ser técnica alternativa ao bloqueio do neuroeixo ou à anestesia geral ${ }^{1,2}$. Estudos recentes demonstram que o bloqueio do nervo ciático é pouco realizado por anestesiologistas, devido à falta de adequado treinamento e por dificuldades técnicas ${ }^{3-5}$.

O nervo ciático é um nervo misto, continuação do fascículo superior do plexo sacral, formado pelas raízes ventrais de $L_{4}$ $\mathrm{a} \mathrm{S}_{3}$, sendo o maior do corpo humano em diâmetro, com cerca de $2 \mathrm{~cm}$ de largura na porção proximal. Sai de pelve através do forâmen isquiático maior, passando abaixo do músculo piriforme, desce entre o trocânter maior do fêmur e a tuberosidade isquiática, e ao longo do dorso da coxa, anterior aos músculos bíceps femoral e semitendinoso, até seu terço inferior, onde se divide em dois grandes ramos denominados nervos tibial e fibular comum.

A anatomia do nervo ciático permite seu acesso por diferentes vias, do espaço parasacral à fossa poplítea, sendo identificado por uma linha imaginária que inicia pela borda inferior do glúteo máximo entre o trocânter maior e a tuberosidade isquiática. Diversas vias de acesso para o bloqueio têm sido descritas. A abordagem por via posterior foi primeiramente descrita por Labat em $1930^{6}$ e posteriormente aperfeiçoada por Winnie ${ }^{7}$. Embora a abordagem posterior seja mais comumente utilizada, tem seu uso limitado em pacientes que necessitam reposicionamento ou nos que apresentam limitação de mobilidade causada por artrite grave, obesidade ou trauma. Em 1959, Ichiyanahi ${ }^{8}$ descreveu a abordagem lateral para o bloqueio do nervo ciático, sendo a atualmente preferida para bloqueio em crianças pela facilidade e segurança da técnica ${ }^{9}$, apesar da necessidade do uso de agulhas longas (acima de $10 \mathrm{~cm}$ ). A abordagem por via anterior foi descrita pela primeira vez em 1963 por Beck ${ }^{10}$, sendo também de difícil execução devido à dificuldade de identificação dos pontos de referência, como a identificação do trocânter maior, especialmente em pacientes idosos, sendo também extremamente dolorosa a identificação nos pacientes com fraturas de membro inferior. Outra limitação à técnica é o uso de agulha longa $(10 \mathrm{~cm})$ para atravessar o trígono femoral, podendo acarretar lesão de vasos femorais, com conseqüente hematoma. Raj e col. em $1975^{11}$ descreveram nova abordagem em posição de litotomia e com novos pontos de referência, permitindo acesso ao nervo ciático com a flexão da coxa sobre o quadril em 90 graus, porém com restrição a pacientes com fratura de colo de fêmur ou portadores de artrite de joelho. Em 1996, Mansour ${ }^{12}$ descreveu a abordagem parasacral com abordagem mais proximal do nervo ciático, sendo a primeira descrição com o paciente em decúbito ventral, utilizando a espinha ilíaca póstero-superior e a tuberosidade isquiática como pontos de referência. Por ser proximal, esta via permite bloqueio do nervo cutâneo posterior da coxa e nervo obturatório. Nova abordagem anterior foi descrita em 1999 por Chelly ${ }^{13}$, que dispensa a identificação do trocânter maior, utilizando como referência a espinha ilíaca ântero-su- perior e o tubérculo púbico, facilitando assim o bloqueio em pacientes com difícil identificação de pontos de referência, necessários a outras abordagens. A desvantagem desta técnica é a profundidade para localização do nervo ( 9,5 a $13,5 \mathrm{~cm}$ ). Recentemente, em 2001 di Benedetto e col. ${ }^{14}$ descreveram nova abordagem com acesso posterior via transglútea.

Como o nervo ciático torna-se superficial na borda inferior do músculo glúteo máximo, no ponto médio do sulco formado na pele entre a nádega e a porção posterior da coxa (sulco glúteo-femoral), o objetivo do trabalho foi descrever nova abordagem posterior para bloqueio do nervo ciático, avaliando as características de identificação do ponto de referência, a qualidade do bloqueio e a satisfação do paciente.

\section{MÉTODO}

O estudo foi aprovado pelo Comitê de Ética Médica do Hospital de Clínicas da Faculdade de Medicina da Universidade Federal de Uberlândia. Após avaliação pré-operatória e esclarecimentos das opções anestésicas foi obtido consentimento esclarecido dos pacientes avaliados. Foram estudados 17 pacientes estado físico ASA I, II ou III, com idades entre 21 e 79 anos, peso de 55 a $90 \mathrm{~kg}$, programados para serem anestesiados com a nova abordagem posterior do bloqueio do nervo ciático e escalados para cirurgia envolvendo perna e pé. Foram excluídos do estudo os pacientes com contra-indicação à anestesia regional, portador de doença psiquiátrica ou abuso de drogas.

Quando o paciente chegou ao centro cirúrgico, foi estabelecida infusão de solução de Ringer com lactato pela inserção de cateter calibre $18 \mathrm{G}$ em veia do antebraço, cerca de $30 \mathrm{mi}-$ nutos antes de encaminhar o paciente à sala de operação. Esta via venosa foi utilizada para que todos os pacientes fossem sedados com midazolam na dose de 1 a $3 \mathrm{mg}$ antes do bloqueio anestésico.

Na sala de cirurgia, a monitorização padrão constou de cardioscópio na derivação $D_{\|}$ou CM5, esfigmomanômetro para controle da pressão arterial pelo método de Riva-Rocci com freqüência de aferição a cada 5 minutos e oxímetro de pulso.

Alguns critérios anatômicos foram considerados para realização do bloqueio. Pela imagem sagital de ressonância eletromagnética (Figura 1) da região proximal da coxa, ao nível do sulco glúteo, observa-se que o nervo ciático é superficial à pele. É interessante notar que neste ponto, a referência cutânea é facilmente identificada pelo sulco entre os músculos semitendinoso e vasto lateral e a inserção do músculo glúteo. É importante assinalar a inexistência de vasos de grande calibre, visto que a artéria femoral encontra-se oposta a esta região.

O paciente foi posicionado em decúbito ventral. Identificado o sulco glúteo-femoral (dobra da pele entre a nádega e região posterior da coxa), utilizou-se o ponto médio deste sulco como ponto de referência para o introdução da agulha a fim 
de realizar o bloqueio (Figura 2). Após anti-sepsia e anestesia com lidocaína a $1 \%$ sem vasoconstritor $(20 \mathrm{mg}$ ), introduziu-se agulha de bisel curto de $5 \mathrm{~cm}$ de comprimento e eletricamente isolada por teflon (Stimuplex A $50^{\circledR}$, B.Braun) conectada a estimulador de nervo periférico (TOF- WATCH ${ }^{\circledR}$ ), introduzida perpendicularmente à pele. A agulha foi introduzida gradativamente com objetivo de atingir o nervo ciático, localizado anteriormente ao músculo semitendinoso, passando pelo sulco delimitado pelos músculos semitendinoso, inserção do glúteo máximo e vasto lateral (Figura 3). O estímulo elétrico aplicado foi inicialmente ajustado com freqüência de $1 \mathrm{~Hz}$ e corrente de 1,0 mA. A uma profundidade de 3 a 5 cm observava-se resposta de estímulo do componente tibial do nervo ciático (inversão do pé e flexão plantar) ou do nervo fibular comum (dorsoflexão ou eversão do pé). Como a movimentação do pé representava resposta motora ao estímulo elétrico, a corrente foi reduzida a $0,5 \mathrm{~mA}$. Caso o movimento parasse, a posição da agulha era ajustada para manter resposta muscular adequada com baixa corrente $(<0,5 \mathrm{~mA})$, considerado ponto ideal para a injeção do anestésico local, sendo utilizado $30 \mathrm{ml}$ de lidocaína a $1 \%$ sem adrenalina (300 $\mathrm{mg}$ ). Não foi pesquisado parestesia. Após dose teste com 2 $\mathrm{ml}$ da solução anestésica, feita para verificar a possibilidade de injeção anestésica intraneural (dor aguda concomitante à injeção anestésica), seguiu-se injeção lenta da dose total, interrompida a cada $5 \mathrm{ml}$, e feita aspiração do êmbolo da seringa para confirmar que a agulha encontrava-se em região extravascular.

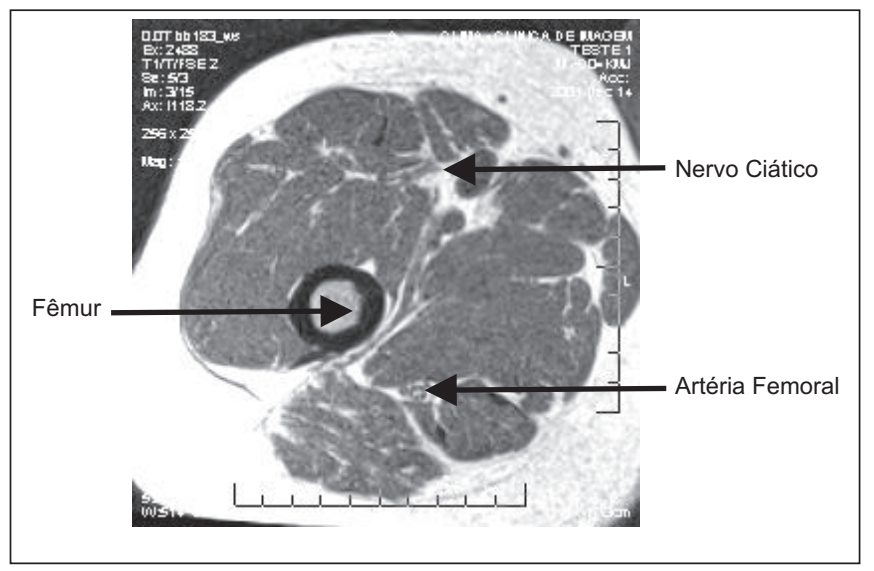

Figura 1 - Imagem de Ressonância Eletromagnética na Região Proximal da Coxa (sulco glúteo)

Todos os bloqueios foram realizados por anestesiologista com experiência em anestesia regional. Nas cirurgias indicadas, foi realizado conjuntamente bloqueio do nervo safeno (ramo sensitivo do nervo femoral) no joelho com $5 \mathrm{ml}$ de lidocaína a $1 \%$.

O bloqueio do nervo femoral foi realizado antes do ciático nos procedimentos cirúrgicos indicados. O paciente foi considerado pronto para o procedimento cirúrgico quando mostrava completa perda da sensação dolorosa à picada de agulha na distribuição do nervo tibial, assegurada pela incapacidade de movimentação efetiva do tornozelo e do pé.

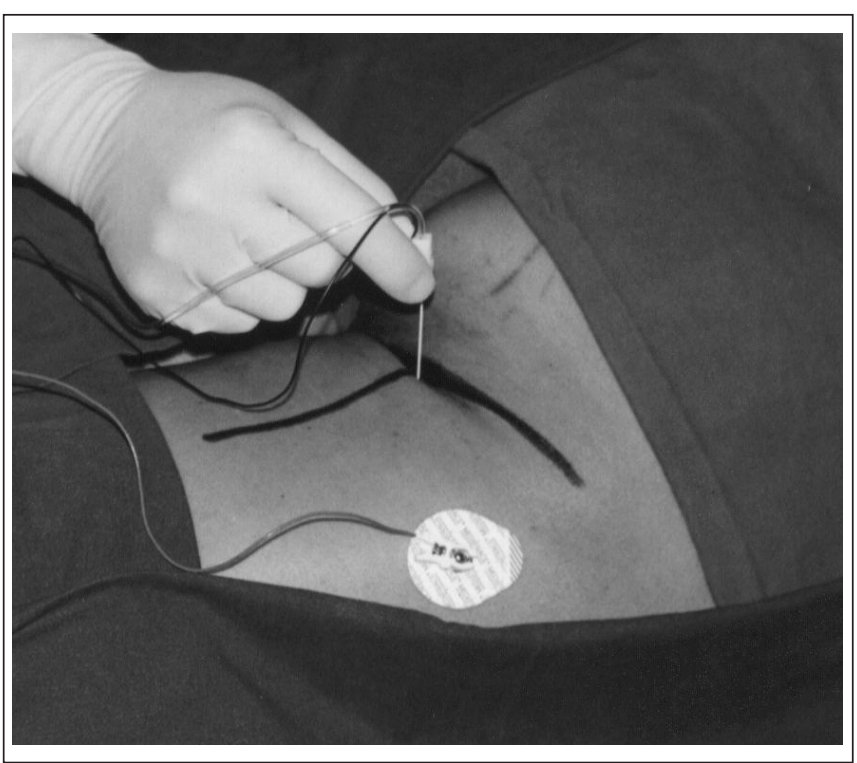

Figura 2 - Referência Anatômica do Sulco Glúteo para Abordagem do Nervo Ciático por Via Posterior Infraglútea

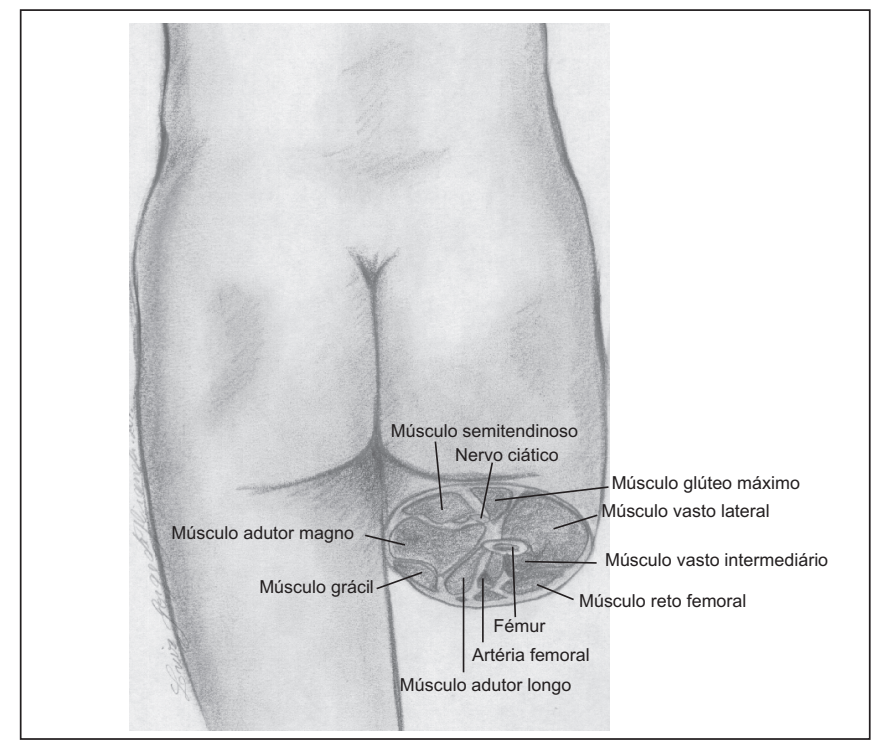

Figura 3 - Vista Posterior com Corte Horizontal Infraglútea da Região Proximal da Coxa

Um observador independente, não envolvido com a realização do bloqueio, observou o tempo entre a introdução da agulha na pele e a resposta motora apropriada ao estímulo elétrico (tempo de realização do bloqueio), tempo entre o fim da administração do anestésico e o momento considerado pronto para o procedimento cirúrgico (latência), número de reposicionamento da agulha necessários à resposta motora desejada, profundidade com o qual o estímulo elétrico ocor- 
reu. Analgesia ou sedação complementar foi relatada pelo mesmo observador independente.

Após o bloqueio, os pacientes foram questionados para graduar a experiência de desconforto durante o procedimento, usando escala de 3 pontos ( 1 = sem dor, 2 = dor moderada, 3 = extremamente doloroso). Em todos os casos a cirurgia foi conduzida sem necessidade complementar de anestesia geral. O bloqueio sensorial foi verificado por aplicação de gelo no dorso (nervo fibular comum) e região plantar do pé (nervo tibial), sendo considerado completo quando o frio não era sentido. O bloqueio motor foi investigado solicitando-se ao paciente a realização de movimento de flexão dorsal e plantar do pé, e considerado completo quando não ocorria movimento. A duração do bloqueio motor e sensorial foi definido como o tempo entre a injeção do anestésico e a recuperação da função motora e sensorial, respectivamente. A satisfação com a técnica anestésica foi avaliada 24 h após o término da cirurgia, usando-se escala de 2 pontos: 1 = satisfeito - "se fosse operar novamente, aceitaria o mesmo tipo de anestesia". 2 = insatisfeito - "se fosse operar novamente, gostaria que fosse realizado outro tipo de anestesia”.

\section{RESULTADOS}

Foi possível localizar o nervo ciático em todos os pacientes sendo o tempo necessário para o estímulo apropriado com $0,5 \mathrm{mAde} 8,58 \mathrm{~min} \pm 5,71$, com profundidade de $3,8 \pm 0,44 \mathrm{~cm}$ após em média de 2 redirecionamentos da agulha, com extremos de 1 a 7 tentativas. Não foram observadas diferenças de estímulo nos ramos tibial e fibular comum do nervo ciático, assim como não foi relatado desconforto para realização do bloqueio. Alatência ao bloqueio sensorial foi de $5,88 \pm 1,6 \mathrm{mi}-$ nutos, sendo completo em todos os casos com o volume e concentração usados. A duração da anestesia foi de 4,05 \pm 1,1 horas (bloqueio sensitivo) e do bloqueio motor de 2,9 \pm 0,8 horas.

Não foram observados aspiração de sangue, parestesias, sinais clínicos de intoxicação anestésica durante ou após a realização da técnica ao bloqueio do nervo ciático. Não ocorreram comprometimentos hemodinâmicos. Não houve deficit sensorial ou motor após a recuperação do bloqueio. As cirurgias realizadas estão na tabela I.

Tabela I - Cirurgias Realizadas nos Pacientes Estudados

\begin{tabular}{lc}
\hline Tipos de cirurgia & $\mathrm{n}=17$ \\
\hline Amputação de dedos do pé & 4 \\
Amputação de pé & 1 \\
Correção de tendão de Aquiles & 1 \\
Correção de varizes na perna & 1 \\
Desbridamento de pé diabético & 5 \\
Fixação de fratura luxação de tornozelo & 1 \\
Retirada de material de síntese de tornozelo & 2 \\
Exérese de tumor em fíbula & 1 \\
Enxerto de pele no tornozelo & 1 \\
\hline
\end{tabular}

\section{DISCUSSÃO}

Diversas e diferentes abordagens ao nervo ciático tem sido descritas na literatura ${ }^{6,9-11,13-15}$, sendo mais utilizada a via posterior clássica descrita por Labat, mas que ainda continua sendo pouco realizado por anestesiologistas, devido à falta de treinamento, dificuldades técnicas e temor às complicações ${ }^{3-5}$, que em geral não estão relacionadas diretamente ao bloqueio. As lesões do nervo ciático decorrente de tentativas de bloqueio são extremamente raras, e as descritas, em geral graves e definitivas, são decorrentes de administração errônea de medicamentos em nádegas de recém-nascidos ${ }^{16-18}$

Estudos experimentais em ratos, com concentrações habituais de anestésicos locais, falharam em demonstrar alterações ou lesões do nervo ciático, mesmo com injeções repetidas ${ }^{19,20}$. Observa-se no máximo, discreto edema endoneural, sem tradução clínica, após utilização de altas concentrações de anestésicos tipo amino-éster, efeito não confirmado com os do tipo amida.

A possibilidade de lesão neurológica é praticamente inexistente quando utilizado material apropriado ao bloqueio, como agulhas eletricamente isoladas, de bisel curto e conectadas a estimulador de nervos periféricos corretamente regulado $^{21,22}$. Autilização do estimulador de nervos periféricos permite conforto ao paciente, dispensando pesquisa de parestesia e necessidade da informação do paciente. Possibilita que seja realizado sob sedação ou com anestesia geral, trazendo satisfação e confiança ao paciente em relação ao procedimento, como mostrou o estudo pelos resultados das tabelas II e III. Outra vantagem é que localiza os nervos periféricos sem provocar lesão mecânica, desde que respeitados os limites de amperagem e que os nervos a serem localizados sejam mistos, pois a pesquisa ao estímulo não é mais a parestesia e, sim, a contração muscular ao estímulo, evitando a possibilidade da neurite traumática.

Apesar do bloqueio do nervo ciático ser pouco realizado na prática clínica, pode ser útil e a melhor opção em alguns pacientes, especialmente naqueles com comprometimento importante do sistema cardiorrespiratório. Praticamente não existem falhas do bloqueio, quando a injeção da solução é feita após a indução de contrações musculares com corrente elétrica menor ou igual a $0,5 \mathrm{~mA}$, como demonstrado neste estudo.

Tabela II - Desconforto Durante a Realização do Bloqueio do Nervo Ciático nos Pacientes Estudados

\begin{tabular}{lc}
\hline Graduação & $\mathrm{n}=17$ \\
\hline 1 & 9 \\
2 & 8 \\
3 & 0 \\
\hline
\end{tabular}


Tabela III - Satisfação dos Pacientes Estudados Quanto ao Bloqueio Realizado

\begin{tabular}{lc}
\hline Graduação & $\mathrm{n}=17$ \\
\hline 1 & 17 \\
2 & 0 \\
\hline
\end{tabular}

Contudo tem sido referida como desconfortável e dolorosa ao paciente, principalmente devido à passagem da espessa camada muscular que a agulha necessita atravessar para atingir regiões próximas ao nervo ciático ${ }^{22}$. Os resultados deste estudo mostram que a nova abordagem posterior pela via do sulco glúteo é efetiva para o bloqueio do nervo ciático, sendo bem tolerado pelo paciente (Tabelas II e III). Utilizando a referência anatômica proposta e estimulador de nervo periférico, o nervo ciático foi identificado em todos os pacientes, sendo efetivo para bloqueio sensorial e motor relacionados, diferente de estudos que mostram falhas de $14 \%$ ao utilizar a via anterior ${ }^{10,23}$

A redução do desconforto da dor durante a realização do bloqueio provavelmente está relacionada à profundidade com a qual o nervo ciático é identificado, descrito com profundidades que variam de 6,7 a $15 \mathrm{~cm}$, dependendo da abordagem utilizada. A redução do desconforto ao bloqueio do ciático observada no presente estudo corrobora estudo prévio em que se comparou a profundidade de introdução da agulha na via posterior transglútea $(4,5 \mathrm{~cm})$ com a via posterior clássica $(6,7 \mathrm{~cm})^{14}$. Este fator, associado com baixa necessidade de redirecionamento da agulha para o sucesso da localização do nervo, provavelmente explicam a aceitabilidade da técnica proposta. Na verdade, Fanelli e col. ${ }^{22}$ mostraram baixa aceitabilidade do paciente às técnicas de injeções múltiplas com auxílio de estimulador de nervo periférico nas diferentes técnicas de bloqueio do nervo ciático em que a identificação dos dois ramos do ciático foram pesquisadas.

A reduzida profundidade com a qual o nervo ciático pode ser atingido pela nova abordagem pode ser valorosa em pacientes obesos, em que o aumento de tecido adiposo na região glútea torna extremamente difícil, ou quase impossível, a realização do bloqueio pela técnica posterior clássica. Essa nova abordagem mostrou-se de fácil execução (tempo médio de execução do bloqueio foi de 5 minutos), sem falhas de bloqueio e possibilidade de se utilizar agulhas de $5 \mathrm{~cm}$ (profundidade média de localização do nervo $4 \mathrm{~cm}$ ). Observou-se facilidade para identificação do ponto de referência, devido à depressão anatômica na região mediana do sulco glúteo-femoral, correspondente à divisão entre os músculos vasto lateral e semitendinoso.

Pela nova técnica proposta não ocorreu anestesia da região correspondente ao nervo cutâneo posterior da coxa, diferentemente da técnica posterior clássica, que ocorre em $90 \%$ dos pacientes. Porém, apresenta vantagens indiscutíveis, como facilidade de identificação do ponto de referência, baixo risco de lesão venosa e impossibilidade de raquianestesia total e peridural como no acesso de Mansoure Bennetts ${ }^{12}$.
O grande diâmetro do nervo ciático pode tornar a latência do bloqueio menos previsível que outros bloqueios periféricos, apesar deste efeito farmacológico ser principalmente influenciado pelas propriedades químicas do anestésico utilizado. Nesta investigação, observou-se latência média de 6 minutos, relativamente menor que estudos prévios que utilizaram outros anestésicos por diferente vias, como a ropivacaína, cuja latência média foi de 10 a 25 minutos $^{13,14,24-26}$, e bupivacaína de 20 a 30 minutos ${ }^{27}$.

Ressalta-se a observação que nenhum paciente necessitou complementação analgésica durante a cirurgia, diferentemente referido por outros autores ${ }^{14}$. O pequeno espaço intermuscular no qual o nervo ciático encontra-se, pela nova técnica descrita, associado com pequena quantidade de tecido conjuntivo envolvendo o nervo, pode favorecer a impregnação do feixe nervoso pelo anestésico local, provavelmente justificando a baixa latência e ausência de suplementação analgésica per-operatória.

Conclui-se, através dos resultados deste estudo prospectivo, que o nervo ciático pode ser facilmente bloqueado pela via posterior descrita. Esta técnica pode ser considerada como alternativa à via posterior clássica de Labat ${ }^{6}$ e a modificada por Winnie ${ }^{7}$.

\section{AGRADECIMENTOS}

Ao Dr. Luciano Martins Messias, médico radiologista da Clínica de Imagens, pelo auxílio no estudo da imagem de ressonância eletromagnética.

\section{Simplified Sciatic Nerve Approach by the Posterior Route at the Median Gluteus-Femoral Sulcus Region, with a Neurostimulator}

Neuber Martins Fonseca, TSA, M.D., Fernando Xavier Ferreira, M.D., Roberto Araújo Ruzi, M.D., Gulherme Carnaval Souza Pereira, M.D.

\section{INTRODUCTION}

The combination of sciatic nerve block and the 3-in-1 technique is satisfactory for patients submitted to lower limb surgeries and may be an alternative to neuraxis blockade or general anesthesia ${ }^{1,2}$. Recent studies have shown that sciatic nerve blockade is seldom performed by anesthesiologists due to lack of adequate training and technical difficulties ${ }^{3-5}$. The sciatic is a mixed nerve, being a prolongation of the upper sacral plexus fascicle, formed by ventral roots from $L_{4}$ to $S_{3}$. It 
is the largest human nerve in diameter, with approximately 2 $\mathrm{cm}$ thick at the proximal region. It leaves the pelvis through the foramen ischiadicum majus passing below the piriform muscle and going down between greater trochanter and ischial tuberosity, continuing along the femoral dorsum, anterior to biceps femoris and semitendinous muscles, until the lower femoral third, where it is divided in two major branches called tibial and common fibular nerves.

The sciatic nerve anatomy allows it to be accessed by different routes, from the parasacral space to the popliteal fossa, over an imaginary line drawn from the inferior gluteus maximus border between greater trochanter and ischial tuberosity. Several access routes have been described. The posterior approach was first described by Labat in $1930^{6}$ and was enhanced by Winnie ${ }^{7}$. Although very popular, the posterior approach has limitations in patients needing repositioning or those with motility constraints caused by severe arthritis, obesity or trauma. In 1959, Ichiyanahi ${ }^{8}$ has described the lateral approach for sciatic nerve block, which is currently preferred for pediatric blockade due to its easiness and safety ${ }^{9}$, in spite of the need for long needles (above $10 \mathrm{~cm}$ ). The anterior approach was first described by Beck in $1963^{10}$ and it is not easy to perform due to difficulties in identifying reference points such as greater trochanter, especially in elderly patients. It is also a painful identification in patients with lower limb fractures. Another limitation is the need of long needles $(10 \mathrm{~cm})$ to cross the femoral trigone, which may injure femoral vessels and cause hematomas. Raj et al. ${ }^{11}$ have described in 1975 a new approach with the patient in the lithotomy position, with other reference points, allowing the access to the sciatic nerve by flexing the thigh 90 degrees over the hip. This approach, however, is limited in patients with femoral neck fracture or those with knee arthritis. Mansour ${ }^{12}$, in 1996, described the parasacral route, a more proximal approach of the sciatic nerve, and was the first to describe patients in the prone position, using the posterior-superior iliac spine and the ischial tuberosity as reference points. For being proximal, this route allows the blockade of the cutaneous femoris posterior nerve and the obturator nerve. A new anterior approach was described by Chelly ${ }^{13}$ in 1999 , which does not identify the greater trochanter, but rather uses as reference the anterior-superior iliac spine and the pubic tubercle, thus helping blockade in patients with difficult identification of reference points required by other approaches. The disadvantage of this technique is the depth of the nerve $(9.5$ to $13.5 \mathrm{~cm})$. Recently, in 2001, Di Benedetto et al. ${ }^{14}$ have described a new posterior transgluteal access approach.

Since the sciatic nerve becomes superficial in the inferior border of the gluteus maximus muscle, at the middle point of the skin fold between the nate and the posterior thigh (gluteus-femoral sulcus), our study aimed at describing a new posterior approach for sciatic nerve block, evaluating reference points identification, blockade quality and patients' satisfaction.

Revista Brasileira de Anestesiologia

Vol. 52, N 6 , Novembro - Dezembro, 2002

\section{METHODS}

The study was approved by the Ethical Committee, Hospital das Clinicas, Faculdade de Medicina, Universidade Federal de Uberlândia. After preoperative evaluation and explanations about the different anesthetic options, patients gave their informed consent. Seventeen patients, physical status ASA I, II or III, aged 21 to 79 years, weighing 55 to $90 \mathrm{~kg}$, scheduled for undergoing foot or leg surgeries were scheduled for the new posterior sciatic nerve blockade. Exclusion criteria were regional anesthesia counterindications, psychiatric patients or drug abuse.

After admission to the operating center, an $18 \mathrm{G}$ catheter was inserted in a forearm vein for lactated Ringer's solution infusion approximately 30 minutes before referral to the operating room. This venous line was used to sedate all patients with 1 to $3 \mathrm{mg}$ midazolam before anesthesia. Standard monitoring consisted of cardioscope in $D_{\|}$or CM5 leads, sphygmomanometer for blood pressure control by the Riva-Rocci method with readings at 5-minute intervals and pulse oximetry.

Some anatomic criteria were considered to perform the blockade. The sagital MRI image (Figure 1) of the proximal thigh at the sulcus gluteus level shows that the sciatic nerve is superficial there. It is interesting to note that, at this point, the skin reference is easily identified by the sulcus formed between semitendinous and vastus lateralis muscles and the gluteus muscle insertion. It is important to stress the inexistence of large vessels, since the femoral artery is opposed to this region.

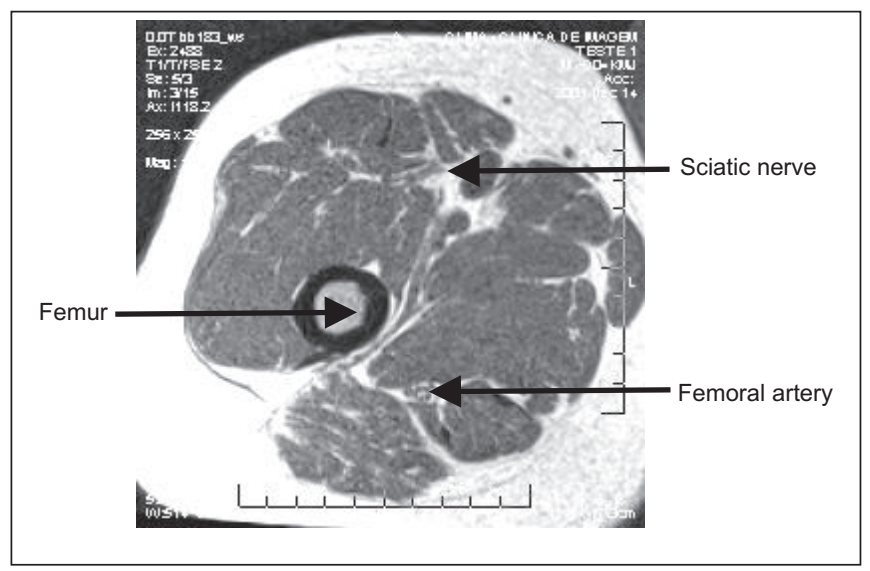

Figure 1 - Electromagnetic Resonance Image of the Proximal Thigh (sulcus gluteus)

Patients were placed in the prone position and the sulcus gluteus (skin fold between nates and posterior thigh) was identified. The middle point of this sulcus was used as the reference point for needle insertion (Figure 2). After skin disinfection and anesthesia with $1 \%$ plain lidocaine $(20 \mathrm{mg})$, a short-bevel $5 \mathrm{~cm}$ needle electrically insulated with teflon 
(Stimuplex a $50^{\circledR}$, B. Braun) connected to a peripheral nerve stimulator (TOF-WATCH ${ }^{\circledR}$ ) was introduced perpendicular to the skin. Needle was gradually introduced aiming at the sciatic nerve located anterior to the semitendinous muscle, crossing the sulcus delimited by the semitendinous muscle and the insertion of the gluteus maximus and the vastus lateralis muscles (Figure 3 ). Electric stimulation was initially adjusted to $1 \mathrm{~Hz}$ frequency and $1.0 \mathrm{~mA}$ current. Stimulation response of the tibial component of the sciatic nerve (foot inversion and plantar flexion) or the common fibular nerve (foot dorsoflexion or eversion) were observed at a depth of 3 to 5 $\mathrm{cm}$. Since foot movements represented a motor response to electric stimulation, current was decreased to $0.5 \mathrm{~mA}$. If movements stopped, needle position was adjusted to maintain adequate muscle response with low current $(<0.5 \mathrm{~mA})$, and this was considered the exact point for the injection of 30 $\mathrm{ml} 1 \%$ plain lidocaine without epinephrine $(300 \mathrm{mg}$ ). Paresthesia was not attempted. After the test dose with $2 \mathrm{ml}$ anesthetic solution to check the possibility of intraneural anesthetic injection (acute pain simultaneously to anesthetic injection), slow total dose injection was started and interrupted at every $5 \mathrm{ml}$, when the syringe was aspirated to confirm that the needle was placed in an extravascular region.

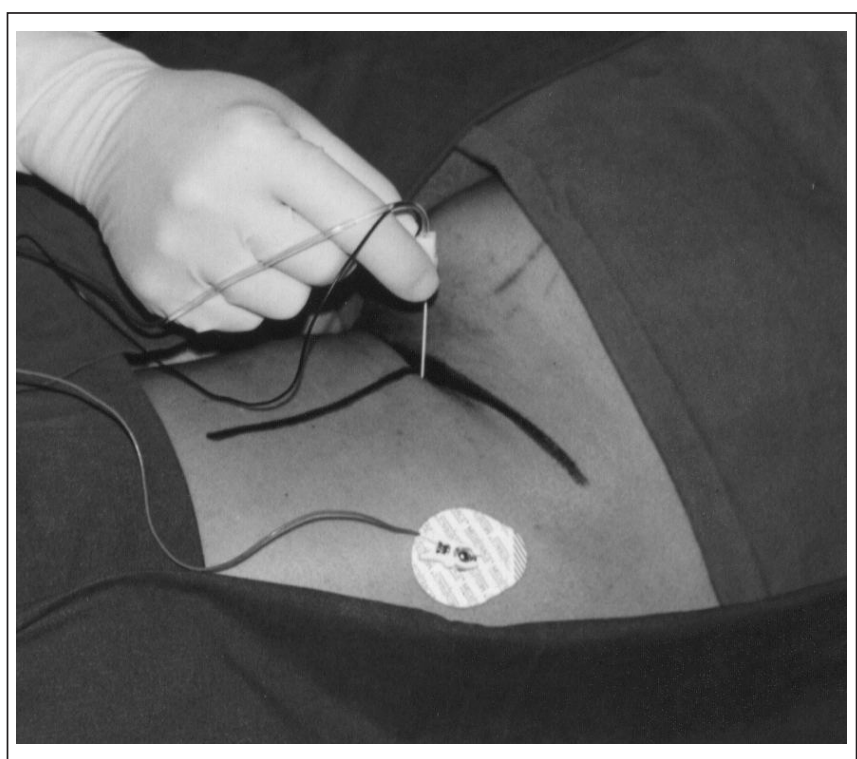

Figure 2 - Sulcus Gluteus Anatomic Reference for Posterior Infragluteal Sciatic Nerve Approach

All blockades were performed by anesthesiologists experienced in regional blocks. If indicated, the saphenous nerve (femoral nerve sensory branch) was also blocked in the knee with $5 \mathrm{ml}$ of $1 \%$ lidocaine.

Femoral nerve was blocked before the sciatic nerve, when indicated for the surgery. Patients were considered ready for the procedure when presenting total loss of pinprick sensation in the tibial nerve distribution area, besides the inability to move ankle and foot.

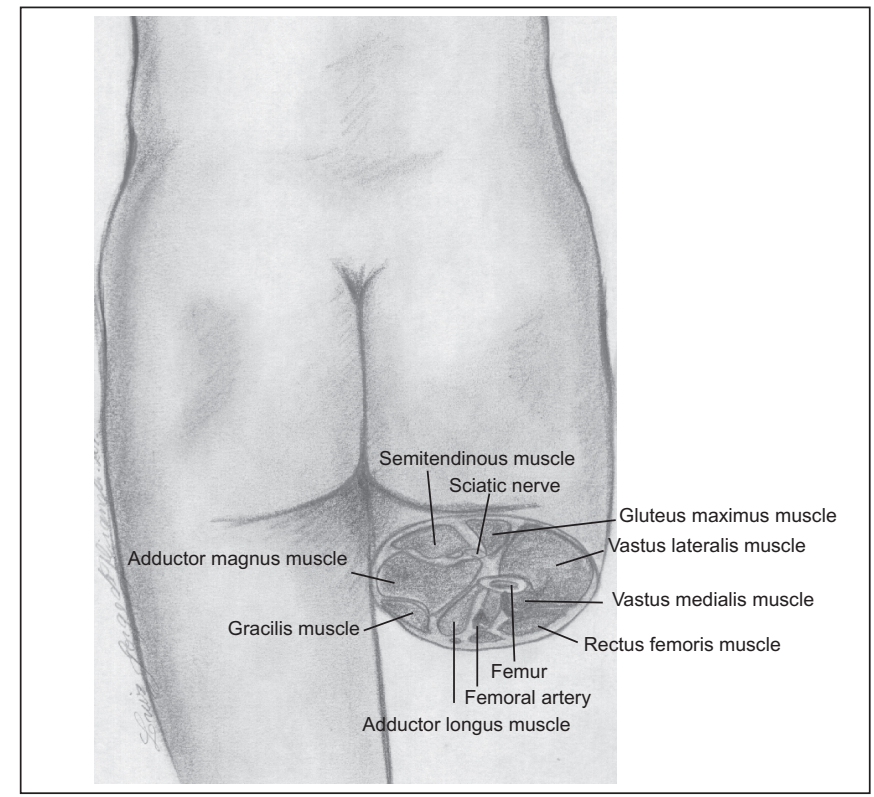

Figure 3 - Posterior View with Horizontal Infragluteal Cross-Section of the Proximal Thigh

An independent observer registered the time elapsed between needle insertion and adequate motor response to electric stimulation (blockade time), time between end of anesthetic administration and patients being considered ready for the surgical procedure (onset time), number of needle repositionings needed for the desired motor response, and needle depth at which there was electric response. Additional analgesia or sedation were reported by the same independent observer.

After blockade, patients were asked to rate their discomfort during the procedure using a 3 -grade scale ( 1 = no pain, $2=$ moderate pain, 3 = extremely painful). There was no need for general anesthesia complementation. Sensory block was evaluated by applying ice on the foot dorsum (common fibular nerve) and plantar region (tibial nerve), and was considered complete when cold was not perceived. Motor block was evaluated by asking patients to flexion the foot and was considered complete in the absence of movement. Motor and sensory block duration was defined as time between anesthetic injection and motor and sensory function recovery, respectively. Satisfaction with the anesthetic technique was evaluated $24 \mathrm{~h}$ after surgery completion using a 2-grade scale: 1 = satisfied - "if I were to be operated on again, I would accept the same anesthesia", 2 = unsatisfied - "if I were to be operated on again, I would prefer a different type of anesthesia".

\section{RESULTS}

The sciatic nerve was identified in all patients and the time needed for adequate stimulation with $0.5 \mathrm{~mA}$ was $8.58 \mathrm{~min} \pm$ $5.71 \mathrm{~min}$, with a depth of $3.8 \pm 0.44 \mathrm{~cm}$ after an average of two needle repositionings, with extremes of 1 to 7 attempts. There were no stimulation differences in the tibial and com- 
mon fibular branches of the sciatic nerve, and no discomfort was reported at blockade. Sensory block onset time was 5.88 \pm 1.6 minutes, and it was complete in all cases with the volumes and concentrations used. Anesthesia duration was $4.05 \pm$ 1.1 hours (sensory block) and $2.9 \pm 0.8$ hours (motor block). There were no blood aspiration, paresthesia or clinical signs of anesthetic intoxication during or after sciatic nerve block. There were no hemodynamic changes, neither sensory or motor deficits after blockade recovery.

Surgeries performed are in table I.

Table I - Surgeries Performed

\begin{tabular}{lc}
\hline Surgeries & $\mathrm{n}=17$ \\
\hline Toes amputation & 4 \\
Foot amputation & 1 \\
Achilles tendon correction & 1 \\
Leg varicose vein correction & 1 \\
Diabetic foot débridement & 5 \\
Ankle fracture/luxation fixation & 1 \\
Ankle synthesis material removal & 2 \\
Fibula tumor excision & 1 \\
Ankle skin graft & 1 \\
\hline
\end{tabular}

\section{DISCUSSION}

Several different sciatic nerve approaches have been described in the literature ${ }^{6,9-11,13-15}$, being the most popular the classic posterior route described by Labat, yet seldom performed by anesthesiologists due to lack of training, technical difficulties and fear of complications ${ }^{3-5}$ which in general are not directly related to the blockade. Sciatic nerve injuries as a consequence of blockade attempts are extremely rare. Those described, in general severe and permanent, are a consequence of wrong drug administration in the nates of newborns ${ }^{16-18}$.

Experimental studies in rats with clinical local anesthetic concentrations have failed to show sciatic nerve changes or injuries, even with repeated injections ${ }^{19,20}$. Nothing more than minor endoneural edema, with no clinical significance, is observed after high concentrations of amino-esther anesthetics, effect yet not confirmed with amides.

There is little chance of neurological injury when adequate material is used, such as short electrically insulated needles connected to an adequately adjusted peripheral nerve stimulator ${ }^{21,22}$. Peripheral nerve stimulation brings comfort to patients with no need for paresthesia search or patients' information. It allows the procedure to be performed under sedation or general anesthesia, bringing satisfaction and confidence to patients, as shown in tables II and III. Another advantage is that it locates peripheral nerves without mechanical injury, provided amperage limits are respected. The nerves to be located must be mixed, since stimulation parameter is no longer paresthesia, rather muscle contraction to stimulation, preventing the possibility of traumatic neuritis.
Table II - Discomfort During Sciatic Nerve Block

\begin{tabular}{lc}
\hline Score & $\mathrm{n}=17$ \\
\hline 1 & 9 \\
2 & 8 \\
3 & 0 \\
\hline
\end{tabular}

Table III - Patients' Satisfaction with Blockade

\begin{tabular}{lc}
\hline Score & $\mathrm{n}=17$ \\
\hline 1 & 17 \\
2 & 0 \\
\hline
\end{tabular}

Although being seldom performed in the clinical practice, sciatic nerve blockade may be useful and the best option for some patients, especially those with major cardiopulmonary involvement. There are virtually no blockade failures when the solution is injected after muscle contractions induction with an electric current equal to or below $0.5 \mathrm{~mA}$, as shown in this study.

The procedure, however, has been described as uncomfortable and painful to patients, especially due to the thick muscle layer the needle has to cross to get close to the sciatic nerve ${ }^{22}$. Our study has shown that the new posterior approach via sulcus gluteus is effective for sciatic nerve block, being well tolerated by the patient (Tables II and III). Using the proposed anatomic reference and a peripheral nerve stimulator, sciatic nerve was identified in all patients and was effective for sensory and motor block, differently from other studies showing $14 \%$ failures by the anterior route ${ }^{10,23}$.

Less discomfort and pain during blockade is probably related to the depth in which the sciatic nerve is identified, which has been described as varying from 6.7 to $15 \mathrm{~cm}$, depending on the approach. Less pain at sciatic nerve block observed in this study confirms a previous study where needle introduction depth in the posterior transgluteal region $(4.5 \mathrm{~cm})$ was compared to the classic posterior route $(6.7 \mathrm{~cm})^{14}$. This fact, associated to less needle redirectioning to successfully identify the nerve, may explain the acceptance of the proposed technique. In fact, Fanelli et al. ${ }^{22}$ have shown a poor acceptance of multiple injection techniques with the aid of a peripheral nerve stimulator in different sciatic nerve block techniques where the identification of both sciatic branches was evaluated. The depth in which the sciatic nerve may be reached by the new approach may be valuable for obese patients, in whom increased gluteal fatty tissue makes extremely difficult, if not impossible, to perform the blockade by the classic posterior technique. This new approach is easy to perform (mean blockade induction time was 5 minutes), with no blockade failures and allows the use of $5 \mathrm{~cm}$ needles (mean depth for nerve location $=4 \mathrm{~cm}$ ). The reference point was easy to identify due to the anatomic depression of the median region of the sulcus gluteus, corresponding to the division between vastus lateralis and semitendinous muscles. 
There has been no cutaneous femoris posterior nerve anesthesia with this new technique, differently from the classic posterior technique where $90 \%$ of patients have this nerve anesthetized. It has, however, clear advantages such as easy reference point identification, low risk for nerve injury and impossibility of total spinal and epidural anesthesia such as with the Mansour and Benetts access ${ }^{12 .}$

The large diameter of the sciatic nerve may lead to a less foreseeable blockade onset time, as compared to other peripheral blocks, although this pharmacological effect is primarily influenced by chemical properties of the anesthetic drug. In our investigation, mean onset time was 6 minutes, relatively lower than previous studies using other anesthetics by different routes, such as ropivacaine (10 to 25 minutes) ${ }^{13,14,24-26}$ and bupivacaine (20 to 30 minutes) ${ }^{27}$.

It has to be stressed that no patient needed analgesic complementation during surgery, differently from what has been reported by other authors ${ }^{14}$. The narrow intermuscular space where the sciatic nerve is inserted, associated to a small amount of connective tissue involving the nerve, may favor the impregnation of the nerve bundle with local anesthetics, probably justifying the short onset time and the lack of perioperative analgesic complementation.

The conclusion of this prospective study is that the sciatic nerve may be easily blocked through this new posterior route. The technique may be an alternative to Labat's ${ }^{6}$ classic posterior route and Winnie's ${ }^{7}$ modified technique.

\section{AKNOWLEDGEMENTS}

We acknowledge Dr. Luciano Martins Mesquita, radiologist, "Clinica de Imagens", for his help in the electromagnetic resonance imaging study.

\section{REFERÊNCIAS - REFERENCES}

01. Winnie AP, Ramamurthy S, Durrani Z - The inguinal paravascular technique of lumbar plexus anesthesia: The "3-in-1" block. Anesth Analg, 1973;52:989-996.

02. Jankowski CJ, Horlocker TT, Rock MJ et al - Femoral 3-in-1 nerve block decreases recovery room time and charges and time to hospital discharge after outpatient knee arthroscopy. Reg Anesth Pain Med, 1998;60:23S

03. Hadzic A, Vloka JD, Kuroda MM et al - The practice of peripheral nerve blocks in the United States: a national survey. Reg Anesth Pain Med, 1998;23:241-246.

04. Bouaziz H, Mercier FJ, Narchi P et al - Survey of regional anesthetic practice among French residents at time of certification. Reg Anesth, 1997;22:218-222.

05. Smith MP, Sprung J, Zura A et al - A survey of exposure to regional anesthesia techniques in American anesthesia residency training programs. Reg Anesth Pain Med, 1999;24:11-16.

06. Labat G - Its Technique and Clinical Applications: Regional Anaesthesia, $2^{\text {nd }}$ Ed, Philadelphia, Saunders, 1924;45-55.
07. Winnie AP - Regional anaesthesia. Surg Clin North Am, 1975;54:861-892.

08. Ichiyanagi K - Sciatic nerve block: lateral approach with patient supine. Anesthesiology, 1959;20:601-604.

09. Dalens B, Tanguy A, Vanneuville G - Sciatic nerve blocks in children: comparison of the posterior, anterior, and lateral approaches in 180 pediatric patients. Anesth Analg, 1990;70: 131-137.

10. Beck GP - Anterior approach to sciatic nerve block. Anesthesiology, 1963;24:222-224.

11. Raj PP, Parks RI, Watson TD et al - A new single-position supine approach to sciatic-femoral nerve block. Anesth Analg, 1975;54:489-493.

12. Mansour NY, Bennetts FE - An observational study of combined continuous lumbar plexus and single-shot sciatic nerve blocks for post-knee surgery analgesia. Reg Anesth, 1996;21:287-291

13. Chelly JE, Delaunay L - A new anterior approach to the sciatic nerve block. Anesthesiology, 1999;91:1655-1660.

14. di Benedetto, Laura B, Andrea $\mathrm{C}$ et al - A new posterior approach to the sciatic nerve block: a prospective, randomized comparison with the classic posterior approach. Anesth Analg, 2001;93:1040-1044.

15. Davies MJ, McGlade DP - One hundred sciatic nerve blocks: A comparison of localization techniques. Anaesth Intensive Care, 1993;21:76-78.

16 - Combes MA, Clark WK, Gergory CF et al - Sciatic nerve injury in infants: recognition and prevention of impairment resulting from intragluteal injections. J Am Med Assoc, 1960;173:1336-1338.

17. Gilles FH, French JH - Postinjection sciatic nerve palsies in infants and children. J Pediatr, 1961;58:195-197.

18. Johnson EW, Raptou AD - A study of intragluteal injection. Arch Phys Med, 1965;46:167-170.

19. Kytta J, Heinonen E, Rosenberg PH et al - Effects of repeated bupivacaine administration on sciatic nerve and surrounding muscle tissue in rats. Acta Anaesthesiol Scand, 1986;30:625-629.

20. Myers RR, Kalichaman MW, Reisner LS et al - Neurotoxicity of local anesthetics: altered perineural permeability, edema, and nerve fiber injury. Anesthesiology, 1986;64:29-35.

21. Smith BE, Allison A - Use of a low-power nerve stimulator during sciatic nerve block. Anaesthesia, 1987;42:296-298.

22. Fanelli G, Casati A, Garancini P et al - Nerve stimulator and multiple injections technique for upper and lower limb blockade: failure rate, patient acceptance and neurologic complications. Anesth Analg, 1999;88:847-852.

23. Manani G, Angel A, Civran E et al - Sciatic nerve block by the anterior and posterior approach for operations on the lower extremity. A comparative study. Acta Anaesthesiol Belg, 1982;3: 183-193.

24. McClellan KJ, Faulds D - Ropivacaine: an update of its use in regional anaesthesia. Drugs, 2000;60:1065-1093.

25. Casati A, Fanelli G, Borghi B et al - Ropivacaine or $2 \%$ mepivacaine for lower limb peripheral nerve blocks. Anesthesiology, 1999;90:1047-1052.

26. Casati A, Fanelli G, Fanelli G et al - Low dose clonidine prolongs postoperative analgesia after sciatic-femoral nerve block with $0.75 \%$ ropivacaine for foot surgery. Anesth Analg, 2000;91: 388-392.

27- Coventry DM, Todd JG - Alkalinisation of bupivacaine for sciatic nerve blockade. Anaesthesia, 1989;44:467-470. 


\section{RESUMEN}

Fonseca NM, Ferreira FX, Ruzi RA, Pereira GCS - Abordaje Simplificado del Nervio Ciático por Vía Posterior, en el Punto Medio del Sulco Glúteo-Femoral, con Uso de Neuroestimulador

Justificativa y Objetivos - El bloqueo del nervio ciático puede ser realizado por varios abordajes con ventajas y desventajas. El nervio ciático es el mayor nervio del cuerpo humano en diámetro y largura. Es la continuación del fascículo superior del plexo sacral $\left(L_{4}, L_{5}, S_{1}, S_{2}\right.$ y $\left.S_{3}\right)$. Sale de la pelvis a través del Fuerone isquiático mayor, pasando por bajo del músculo piriforme, baja entre el trocanter mayor del femur y la tuberosidad isquiática, y al largo del dorso del muslo, anterior a los músculos bíceps femoral y semitendinoso, hasta el tercero inferior del muslo, donde se divide en dos grandes ramos denominados nervios tibial y fibular común. Se torna superficial en la borda inferior del músculo glúteo máximo. Baseados en esta descripción anatómica, desenvolvemos un abordaje posterior, teniendo como ventajas la identificación fácil de la anatomía de superficie, superficialidad del nervio en esta localización, provocando menor incomodidad al paciente que otros abordajes y pudiendo utilizar aguja de $5 \mathrm{~cm}$.
Método - Fueron estudiados 17 pacientes, estado físico ASA I, II ó III, con edades entre 21 y 79 años, peso de 55 a $90 \mathrm{~kg}$, sometidos a cirugías en piernas o pies. Después de monitorización, el paciente fue posicionado en decúbito ventral y realizado bloqueo en el punto medio del sulco glúteo femoral (dobla de la piel entre la nalga y la región posterior del muslo), con auxilio de neuroestimulador, utilizando lidocaína a 1\% sin adrenalina (300 mg). Se evaluó la latencia, tiempo de ejecución del bloqueo, anestesia de los nervios tibial, fibular común y cutáneo posterior del muslo. Cuando necesario, fue también realizado el bloqueo del nervio safeno con $5 \mathrm{ml}$ de lidocaína a $1 \%$.

Resultados - Se obtuvo anestesia adecuada en todos los casos con el volumen y concentración usados. En ningún paciente ocurrió anestesia del nervio cutáneo posterior del muslo. El tiempo de ejecución del bloqueo fue de 8,58 $\pm 5,71$ min. la latencia fue de 5,88 $\pm 1,6$ min. La duración sensitiva y motora del bloqueo fue de 4,05 $\pm 1,1$ y 2,9 \pm 0,8 horas, respectivamente.

Conclusiones - Ese nuevo abordaje es eficaz y de fácil ejecución. No está indicada si el bloqueo del nervio cutáneo posterior del muslo es necesario. 